Ann. Biol. anim. Bioch. Biophys., 1978, 18 (6), 1443-1453.

\title{
Etude de l'incorporation des acides gras alimentaires dans les lipides du lait : influence de l'acide phytanique
}

\author{
par R. F. GLASCOCK, V. A. WELCH
}

National Instifute for Research in Dairying.

Shinfield, Reading, RG2 9AT, England.

Summary. Study of dietary fatty acid incorporation in milk lipids. Influence of phytanic acid.

We usually measure the proportion of milk fat derived from the blood precursor by giving a labelled fatty acid to a cow and comparing the mean specific radioactivities of precursor and product during the following week. To test the validity of this method we gave a mixture of stearic and palmitic acids labelled with ${ }^{14} \mathrm{C}$ and ${ }^{3} \mathrm{H}$ to lactating cows and studied the transfer of the two nuclides to blood and milk lipids.

The nuclide ratios in precursor and product, initially similar, evolved differently. We found this to be due in part to the progressive replacement of stearic acid in precursor triglycerides by phytanic acid which, however, was not transferred to milk. When we used mean ${ }^{14} \mathrm{C}$ specific radioactivities of phyłanate-free triglyceride molecules in the calculations we obtained the same value for the proportion of milk fat derived from the precursor as that obtained from the ${ }^{3} \mathrm{H}$ measurements.

By means of a model set up and tested on a computer we showed that even when there is no fractionation of fatty acids the nuclide ratio can be changed by an influx of unlabelled molecules such as would have occurred in the udder as palmitate was formed from acetate.

We discuss the cause of the increasing content of phytanic acid in the precursor triglycerides and suggest that it may have been a reaction to stress. We conclude that our method of measuring the contribution of blood triglycerides to milk fat is valid provided that a labelled acid other than stearic acid is used.

Il est bien connu que les lipides du lait trouvent leur origine en partie dans la synthèse intramammaire à partir de petites molécules et en partie dans un précurseur lipidique circulant dans le sang. Nous avons nous-mêmes identifié ce précurseur comme la fraction triglycéridique des lipoprotéines précipitables au sulfate de dextrane (les lipoprotéines précipitables, LPP) (Glascock ef al., 1966), dont celle trouvée dans le sang veineux mammaire peut être considérée comme un échantillon. Pour calculer la proportion des lipides du lait provenant de ces triglycérides nous avons utilisé le rapport entre les radioactivités spécifiques moyennes du précurseur et des lipides du lait dans les jours qui suivent l'introduction d'un acide gras marqué dans le rumen (Glascock ef al., 1966 ; Bishop ef al., 1969). Comme la radioactivité de ces lipides est presque entièrement redevable à un seul acide marqué il est évident que notre calcul n'est valable que si tous les acides gras renfermés dans les triglycérides précurseurs s'incorporent dans les lipides du lait dans les mêmes proportions qu'ils se présentent 
à la glande mammaire, c'est-à-dire sans fractionnement. Ainsi est notre hypothèse de départ que la prudence nous a incités à vérifier. Pour ce faire nous avons étudié le méłabolisme de deux acides gras différents, marqués respectivement au ${ }^{3} \mathrm{H}$ et au ${ }^{14} \mathrm{C}$ et administrés simultanément à l'animal expérimental.

\section{Matériel et méthodes.}

Acides gras marqués. - L'acide $\left[{ }^{1-14} \mathrm{C}\right]$ stéarique est acheté au Radiochemical Centre, Amersham (Angleterre) et l'acide $\left[9,10^{3} \mathrm{H}\right]$ palmitique est préparé selon la méthode de Glascock el Reinius (1956) à partir du palmitoléate de méthyle. On s'est assuré de la pureté de ces produits en les chromatographiant sur colonne de trisilicate de magnésium.

Préparation des émulsions. - Des quantités d'acides gras marqués renfermant pour chaque expérience les radioactivités indiquées dans le tableau 1 sont mis en émulsion dans $50 \mathrm{ml}$ d'eau selon Glascock et al. (1966).

Animaux. - Nous avons réalisé les expériences chez 3 vaches de race Frisonne de l'élevage de l'Institut. Elles sonf mises en stabulation avant la réalisation des expériences pour les habituer aux conditions et régimes expérimentaux. Chaque vache reçoit une ration composée de foin ef de concentré en quantités approximativement proportionnées à sa production laitière. On administre la dose d'émulsion radioactive par la voie d'une canule de rumen et on prélève les échantillons de sang à l'aide des canules préalablement introduites dans les veines jugulaires et mammaires. Immédiatement après chaque prise de sang aux heures indiquées sur les figures on injecte l'ocytocine et on trait l'animal à la machine. Toutes les conditions pour les 2 expériences définitives sont résumées dans le tableau 1.

TABLEAU 1

Expériences 2 et 3. Résumé des conditions expérimentales

\begin{tabular}{|c|c|c|}
\hline & 2 & 3 \\
\hline 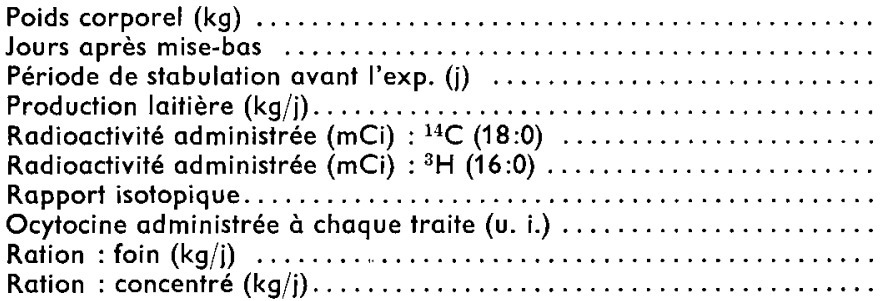 & $\begin{array}{l}560 \\
232 \\
14 \\
13,0 \\
2,00 \\
9,4 \\
0,213 \\
10 \\
8,2 \\
6,4\end{array}$ & $\begin{array}{l}582 \\
218 \\
50 \\
15,5 \\
1,76 \\
77,6 \\
0,0227 \\
2 \\
9,1 \\
7,3\end{array}$ \\
\hline
\end{tabular}

Isolement des lipoprotéines ef extraction des triglycérides. - Les LPP sont isolées par l'emploi du sulfate de dextrane (Glascock ef al., 1966) et dans l'expérience 3 elles sont sous-fractionnées par ultracentrifugation selon Brumby ef Welch (1970) en 
LDL $_{2}^{*}$ (LDL* étant absente) et VLDL*. Les lipides mixtes des lipoprotéines sont extraits selon Glascock ef al. (1966) ef les triglycérides sont séparés par chromatographie sur couche mince selon Bishop ef al. (1969). Un échantillon de $50 \mathrm{ml}$ environ du lait de chaque traite est lyophylisé et les lipides sont extraits par la technique de Glascock et al. (1966).

Séparation chromatographique en phase gazeuse (CPG) des acides gras. - Les acides gras renfermés dans les différents lipides sont transformés en esters méthyliques et séparés dans un appareil Pye 104 essentiellement selon les indications de Stead et Welch (1975) et de Glascock et Welch (1974).

Mesures de radioactivité. - Les radioactivités ${ }^{3} \mathrm{H}$ ef ${ }^{14} \mathrm{C}$ des échantillons sont mesurées dans un spectromètre à scintillation liquide selon Bishop ef al. (1971) ef les radioactivités spécifiques sont obtenues à partir d'un dosage gravimétrique (lait), ou CPG avec étalon interne (lipides lipoprotéiques).

Rapports isotopiques. - Pour faciliter la comparaison des résultats nous avons normalisé le rapport isotopique dans les différents lipides en divisant les rapports observés par celui dans la dose injectée (tabl. 1).

Fraction du produit provenant du précurseur. - Les surfaces délimitées par l'abscisse et les courbes de radioactivité spécifique en fonction du temps des triglycérides précurseurs et des lipides du lait traduisent les radioactivités spécifiques moyennes de ces deux substances pour la durée de l'expérience. Le rapport entre ces radioactivités moyennes donne la contribution quantitative du précurseur au produit (Bishop ef al., 1969).

\section{Résultats.}

Répartition des isotopes dans les lipides. - L'analyse radio-CPG montre que la presque totalité de chaque isotope renfermé dans les triglycérides du précurseur et du lait est liée aux acides de même longueur de chaîne que ceux dans la dose. II ne s'est donc pas produit de recyclage significatif des isotopes.

Expérience no 1. - Dans une expérience préliminaire les lipides des différents échantillons de lait obtenus avec et sans l'usage de l'ocytocine ont la même composition en acides gras, mettant ainsi en évidence que cette hormone ne modifie en rien le métabolisme lipidique du pis. Nous avons donc continué l'usage de l'ocytocine dans les expériences 2 et 3 . Cette expérience, dans laquelle nous avons pris de nombreux échantillons de lait mais seulement 3 échantillons de sang montre aussi que le rapport isotopique moyen dans les triglycérides des LPP pendant la période 17-26 h est inférieur à celui dans la dose injectée $(0,920$ contre 1,000) mais égal à celui dans les lipides du lait. Les résultats suggèrent néanmoins que la courbe des triglycérides aurait évolué de façon toute différente de celle des lipides du lait et dans les expériences 2 et 3 nous avons donc pris un plus grand nombre d'échantillons de sang.

* Dénominations des lipoprotéines: $\operatorname{LDL}_{1}$ : lipoprotéine légère $n^{0} 1(1,019<d<1,039)$; $\mathrm{LDL}_{2}$ : lipoprotéine légère $n^{0} 2(1,039<d<1,060) ; \mathrm{VLDL}$ : lipoprotéine très légère $(d<1,019)$. 
Expérience $n^{\circ}$ 2. - On voit sur la figure 1 l'évolution du rapport isotopique dans les lipides du lait et dans les triglycérides des LPP sanguins provenant des veines jugulaires et mammaires respectivement. Pour des raisons exposées ailleurs (Charton et al., 1965 ; Bishop ef al., 1969) nous considérons le sang jugulaire comme équivalent, aux fins de ces expériences, au sang artériel. Nous utilisons donc le terme commode "différence arteroveineuse " (AV) bien qu'en réalité toutes les mesures dites artérielles aient été effectuées sur du sang jugulaire.

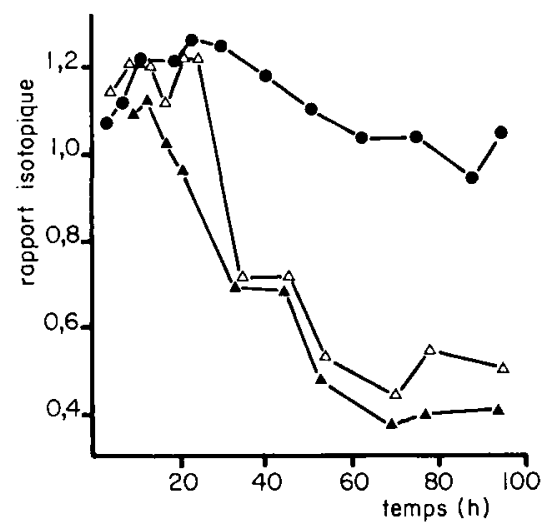

FIG. 1. - Evolution du rapport isotopique (Exp. 2). - Lipides du lait.

$\triangle$ Triglycérides des lipoprotéines précipitables (LPP) jugulaires. $\triangle$ Triglycérides des LPP mammaires.

La radioactivité retrouvée dans trois échantillons des triglycérides de l'expérience 1 nous a fait soupçonner que le rapport isotopique a été presque toujours inférieur à celui des lipides du lait et dans cette $2^{2}$ expérience on voit qu'en effet il en est ainsi (fig. 1). On note cependant qu'il y a une différence AV appréciable dans le rapport.

On trouve une explication, au moins partielle, à cette diminution du rapport isotopique dans les variations de composition en acides gras des triglycérides (fig. $2 a$ ). Tandis que le taux du 16:0 reste presque constant celui du 18:0 diminue progressivement. Il en résulte que le rapport entre les teneurs en ces deux acides (18:0/16:0) évolue à peu près de la même manière que le rapport isotopique pour atteindre cependant une valeur finale relativement moins basse. La perte en 18:0 est compensée par une augmentation corrélative en acide 3, 7,11, 15-tétraméthylhexadecan-1-oïque (acide phytanique, 20:0 $0_{\mathrm{ra}}$; voir aussi Stead et Welch, 1975) de sorte que la somme de leurs concentrations dans les triglycérides reste presque constante.

On n'observe de différence AV constante dans la composition des triglycérides précurseurs que dans les taux de ces deux acides : le taux du 18:0 diminue et celui du 20:0 ra augmente pendant le passage du sang à travers la mamelle. Il en résulte qu'on observe aussi une différence $A V$ dans le rapport 18:0/16:0 comme dans le rapport isotopique (voir plus haut).

Contrastant avec la composition variable des triglycérides lipoprotéiques celle des lipides du lait reste remarquablement constante (fig. $2 b$ ), et en particulier on note l'absence presque complète du 20:0 ra. 
La figure 3 montre l'évolution en fonction du temps des radioactivités spécifiques ${ }^{14} \mathrm{C}$ (lié à 18:0) ef ${ }^{3} \mathrm{H}(16: 0)$ des lipides du lait et des triglycérides mixtes des LPP veineuses mammaires. Les rapports des surfaces délimitées par l'abscisse et les courbes du lait ef des triglycérides sont de 0,638 pour le ${ }^{14} \mathrm{C}$ et de 0,437 pour le ${ }^{3} \mathrm{H}$.

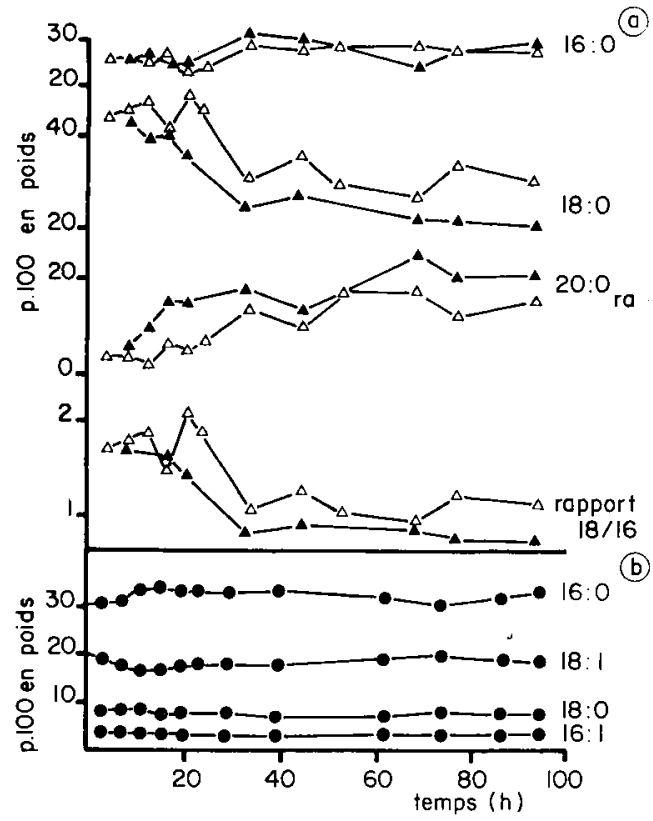

FIG. 2. - Expérience 2. Teneurs en acides gras des lipides (a) du sérum sanguin ef (b) du lait. $\triangle$ Triglycérides des LPP jugulaires. ^ Triglycérides des LPP mammaires. • Lipides du lait.
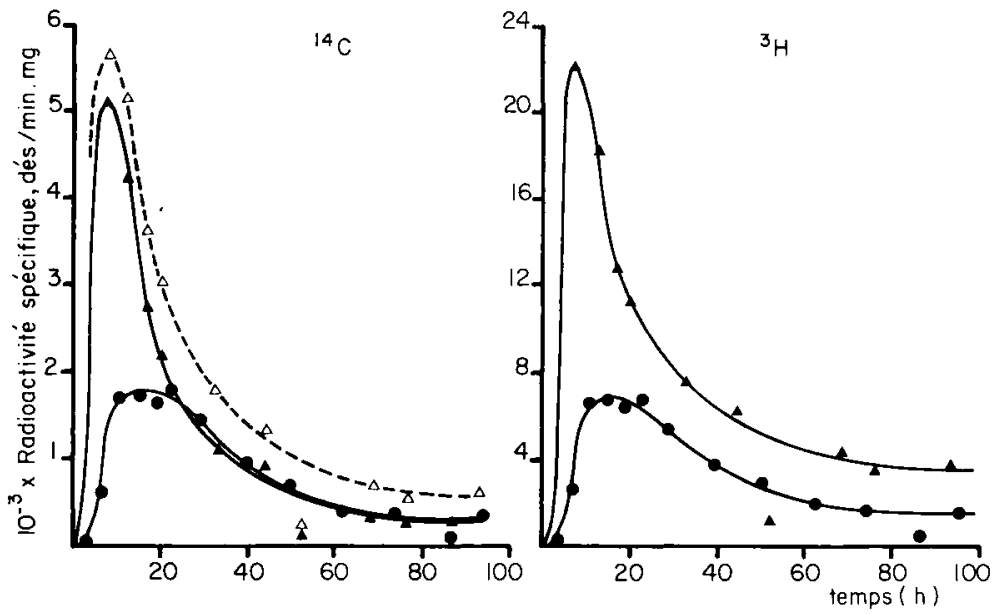

FIG. 3. - Expérience 2. Evolution des radioactivités spécifiques ${ }^{14} \mathrm{C}$ ef ${ }^{3} \mathrm{H}$.

- Lipides du lait. $\Delta$ Triglycérides des LPP mammaires. $\triangle$ Valeurs corrigées (voir texte). 
Des courbes analogues ont été obtenues pour les acides $18: 0+18: 1$ et 16:0 respectivement ef les rapports de surfaces sont de 1,068 pour $18: 0+18: 1$ et de 0,341 pour 16:0.

Le désaccord entre les résultats pour les triglycérides est trop important pour être dû à l'erreur expérimentale. Par contre, les valeurs calculées pour les acides individuels sont bien plus plausibles, les rapports des surfaces (voir plus haut) indiquant que 106,8 p. 100 des acides 18:0 + 18:1 des lipides du lait proviennent des lipoprotéines précipitables contre 34,1 p. 100 pour le 16:0. II esł clair cependant que la valeur pour les acides en $C_{18}$ est entachée d'une erreur de 7 p. 100 environ.

Répartition des acides gras dans les triglycérides. - L'analyse CPG des triglycérides des LPP ef des monoglycérides résultani d'une digestion partielle à la lipase pancréatique selon Stead et Welch (1975) indique qu'à l'exception du 18:1 les principaux acides gras présentent une spécificité de position marquée : $60 \mathrm{p}$. $100 \mathrm{du}$ 16:0 sont localisés en position 2 tandis que la presque totalité du 20:0 ra est fixée en position externe. Le 18:0 exhibe aussi une forte préférence pour les positions externes, mais dans ce cas on observe aussi une différence $A V$ statistiquement significative $(P<0,05)$ dans la répartition de l'acide : 87,0 p. 100 en position externe dans les triglycérides jugulaires et 80,0 p. 100 dans les friglycérides veineux mammaires.

Le remplacement progressif et préférentiel du 18:0 par le 20:0 ra ajouté à la constance de composition des lipides du lait malgré une composition très variable dans son précurseur présumé, nous ont conduits à émettre l'hypothèse que les triglycérides envahis par le 20:0 $\mathrm{ra}_{\mathrm{a}}$ ne servent pas de précurseur des lipides du lait. Cela aurait pour résultat une sous-estimation de la radioactivité spécifique ${ }^{14} \mathrm{C}$ du vrai précurseur sans affecter pour autant les données ${ }^{3} \mathrm{H}$ car les molécules en cause ne sont déficitaires qu'en 18:0.

On peut calculer la radioactivité spécifique, $S_{\mathrm{ex}}$, des molécules exemptes de $20: 0_{\mathrm{ra}}$ par la formule:

$$
\mathrm{S}_{\mathrm{ex}}=\mathrm{S}_{\mathrm{obs}} \times \frac{\left[20: 0_{\mathrm{ra}}\right]+[18: 0]}{[18: 0]}
$$

où $S_{\text {obs }}$ est la radioactivité spécifique observée des triglycérides ef les termes entre crochets sont les taux moléculaires des acides dans les triglycérides.

Appliquée aux radioactivités spécifiques ${ }^{14} \mathrm{C}$ des triglycérides des LPP de l'expérience 2 cette formule donne une nouvelle série de valeurs représentées sur la figure 3 par la courbe en pointillé. Le rapport des surfaces de la courbe du lait ef de celle-là est maintenant de 0,456 , valeur voisine de celle des courbes ${ }^{3} \mathrm{H}$.

Expérience 3. - Les LPP sont généralement séparables en 3 sous-fractions : VLDL, $L D L_{1}$ ef $L D L_{2}$ dont les $\mathrm{LDL}_{1}$ sont parfois absentes chez certaines vaches (Brumby et Welch, 1970 et résultats non publiés). Lorsque le 20:0 ra est présent dans les triglycérides des LPP, ceux des VLDL en sont presque exempts tandis que cet acide atteint parfois une haute concentration dans les triglycérides des $L L_{1}$ ef $L D L_{2}$ (Stead ef Welch, 1975). Nous avons démontré dans un travail précédent (Glascock ef Welch, 1974) que 75 p. 100 des acides gras lipoprotéiques incorporés dans les lipides du lait proviennent des VLDL et 25 p. 100 proviennent des $L L_{1}$ (les $L D L_{2}$ ne faisant pas une contribution significative). Ces constatations nous ont fourni le moyen d'étudier 
l'évolution de la radioactivité spécifique des triglycérides précurseurs des lipides du lait exempts de 20:0 ra.

Pour effectuer l'expérience 3 nous avons donc choisi une vache dont le sang était exempt de $\mathrm{LDL}_{1}$. Ainsi, des triglycérides lipoprotéiques présents, seuls ceux renfermés par les VLDL faisaient fonction de précurseur des lipides du lait. Cette expérience montre que dans ces conditions les radioactivités spécifiques ${ }^{14} \mathrm{C}$ et ${ }^{3} \mathrm{H}$ normalisées sont telles que les courbes sont superposables tant pour les triglycérides des VLDL (fig. 4a) que pour les lipides du lait (fig. 4b). Les rapports des surfaces délimitées par les courbes du lait ef des triglycérides sont donc très voisins pour les deux isotopes, à savoir 0,235 pour le ${ }^{14} \mathrm{C}$ et 0.213 pour le ${ }^{3} \mathrm{H}$.

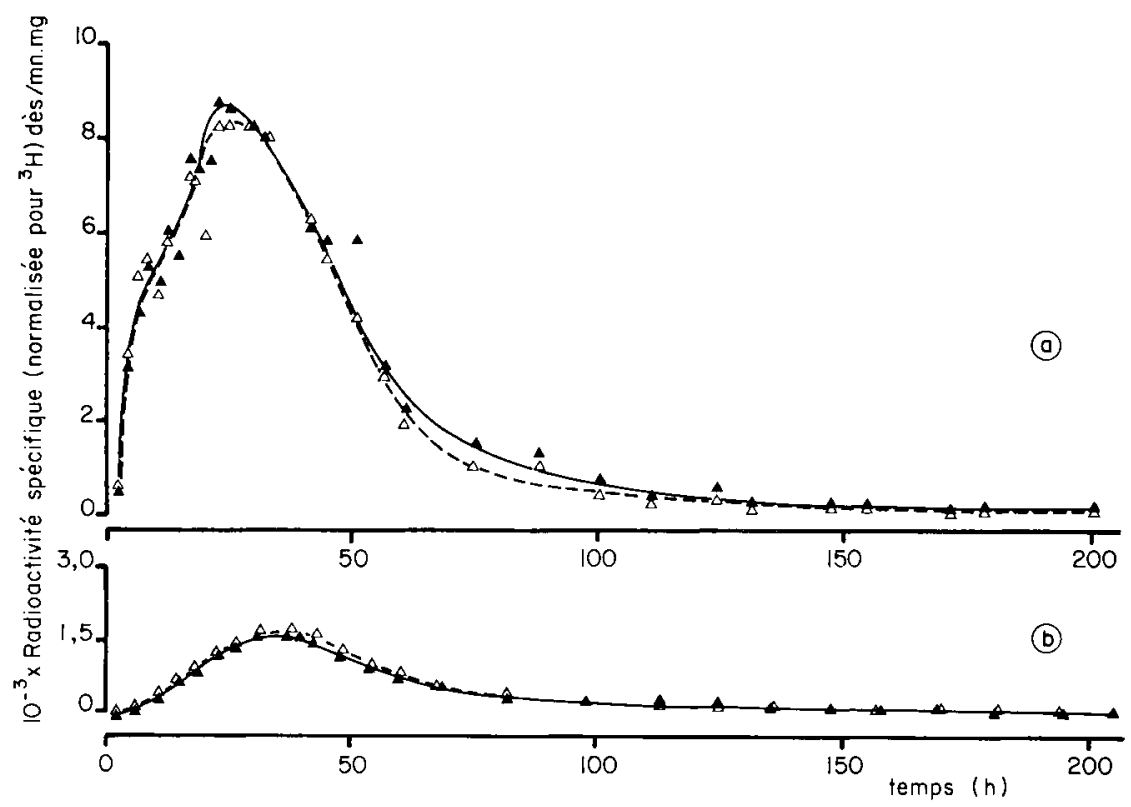

FIG. 4. - Expérience 3. Evolution des radioactivités spécifiques ${ }^{14} \mathrm{C}$ ef ${ }^{3} \mathrm{H}$.

(a) Triglycérides des VLDL mammaires; (b) Lipides du lait. $\triangle{ }^{14} \mathrm{C} . \triangle{ }^{3} \mathrm{H}$.

La comparaison des surfaces délimitées par les courbes de radioactivité spécifique en fonction du temps des acides 16:0 ( $\left.{ }^{3} \mathrm{H}\right)$ et 18:0 $+18: 1\left({ }^{14} \mathrm{C}\right)$ dans le lait à celles des mêmes acides dans les triglycérides lipoprotéiques donne 18,3 p. 100 et 58,1 p. 100 respectivement comme la proportion de ces acides dans ces lipides du laif provenant des mêmes acides dans les triglycérides des VLDL.

La figure 5 montre l'évolution du rapport isotopique dans les triglycérides des VLDL et dans les lipides du lait. Dans le premier le rapport est très variable et à partir de $56 \mathrm{~h}$ est bien inférieur à l'unité. Par contraste le rapport dans les lipides du laif évolue de façon toute différente et est toujours supérieur à celui de son précurseur sanguin.

Nous trouvons aussi que les feneurs des triglycérides des VLDL en acides 16:0 ef 18:0, quoique variables, ne montrent aucune tendance définitive et, ce qui contraste 
avec le rapport isotopique, il en est de même pour le rapport 18:0/16:0. Dans les triglycérides des $\mathrm{LDL}_{2}$ l'évolution des teneurs en acides $16: 0,18: 0$ et $20: 0_{\text {ra }}$ est analogue à celle observée pour ces acides dans les triglycérides des LPP de l'expérience 2 ; tandis que la teneur en 16:0 reste plus ou moins constante la teneur en 18:0 diminue de 40 à 17 p. 100 à mesure que celle en 20:0 ra augmente de 3 à 18 p. 100 .

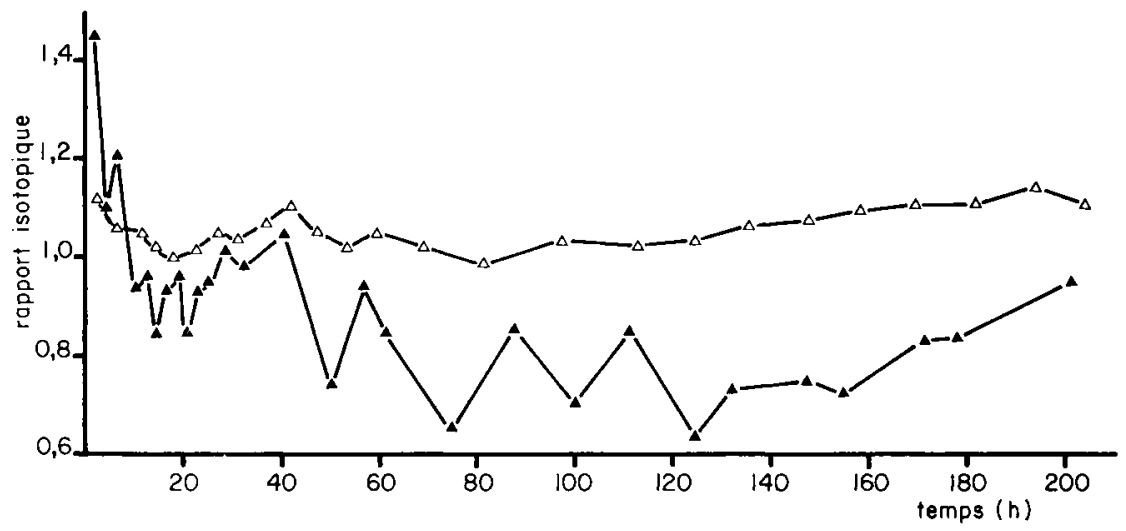

FIG. 5. - Expérience 3. Evolution du rapport isotopique. $\triangle$ Lipides du lait. A VLDL mammaires.

\section{Discussion.}

Les valeurs pour la proportion des lipides du lait provenant du précurseur sérique ef calculées à partir des données ${ }^{3} \mathrm{H}$ et ${ }^{14} \mathrm{C}$ concordent assez bien lorsqu'on exclut soit mathématiquement (exp. 2) soit physiquement (exp. 3) les molécules porteuses de 20:0 $0_{\text {ra }}$ ef sans la faible teneur (3 p. 100 environ) en cet acide enregistrée pour les VLDL (exp. 3) on s'attendrait à voir une concordance encore plus étroite. Ce résultat implique qu'à l'exception du 20:0 ra tous les acides gras renfermés dans les triglycérides des VLDL et $\mathrm{LDL}_{1}$ sont utilisés proportionnellement à leurs taux dans les précurseurs. Cette conclusion semble cependant être contredite par l'évolution des rapports isotopiques : le rapport dans les lipides du lait croît plus ou moins régulièrement tandis que celui dans les triglycérides des VLDL reste voisin, du moins en moyenne, de l'unité jusqu'à 56 h, puis diminue. Comme la majeure partie de la radioactivité retrouvée dans le lait a éłé sécrétée avant ce temps, il est possible qu'une utilisation différentielle des deux acides marqués se soit produite trop tard pour influencer de façon significative les quantités totales des deux isotopes sécrétées dans le lait, car à partir de $56 \mathrm{~h}$ environ les radioactivités sont très faibles.

Comme un déséquilibre nutritionnel n'est guère concevable chez un animal soumis à deux semaines de régime de stabulation on doit se demander si les procédés expérimentaux pourraient provoquer un fractionnement. Le stress est connu pour avoir nombre d'effets biochimiques disparates et on sait que les acides 16:0 et 18:0 n'ont pas exactement le même comportement biochimique : par exemple ils sont repartis différemment entre les trois positions de la molécule triglycéridique. Néanmoins leurs propriétés sont si voisines que l'hypothèse d'un fractionnement tardif provoqué par le stress est très difficilement acceptable. 
Nous avons donc cherché une autre explication dans les effets de la biosynthèse intramammaire à partir d'acétate. Cette contribution endogène est normalement de 60 p. 100 pour l'acide 16:0 du lait et nulle, ou presque nulle, pour les acides 18:0 + 18:1. Il en est ainsi dans l'expérience 2 mais dans l'expérience 3, où les résultats sont plutôt anormaux, les chiffres sont 82 et 42 p. 100 respectivement. II y a cependant, toujours une forte différence entre les apports d'acides endogènes en $C_{16}$ et en $C_{18}$ et donc entre les degrés de dilution des deux acides marqués par les acides non-marqués. Nous nous sommes demandé si cette dilution différentielle pourrait être à l'origine de l'évolution du rapport isotopique dans le lait.

Dans une tentative de vérification de cette hypothèse nous avons conçu le modèle représenté dans la figure 6 . Chaque paire de compartiments correspond à la masse tołale des acides triglycéridiques en $C_{16}$ et $C_{18}$ aux niveaux respectivement des lipides alimentaires, des VLDL et des lipides mammaires. Les valeurs des paramètres correspondent approximativement à celles observées ou calculées dans l'expérience 3 ef les flux sont ajustés de façon que la proportion du contenu des compartiments I et II qui passe par unité de temps au compartiment suivant soit la même pour chaque acide : c'est-à-dire qu'il n'y ait pas de fractionnement. Seuls les efflux de III ne remplissent pas cette condition à cause de l'influx des acides endogènes.

Programmé sur ordinateur, ce modèle démontre qu'après l'introduction de ${ }^{14} \mathrm{C}$ et de ${ }^{3} \mathrm{H}$ en quantités égales dans la première paire de compartiments, le rapport isotopique dans l'efflux de la IIle paire augmente régulièrement (fig. 6). Par contre ceux dans l ef II restent égaux à l'unité. II paraît donc que la dilution différentielle des acides
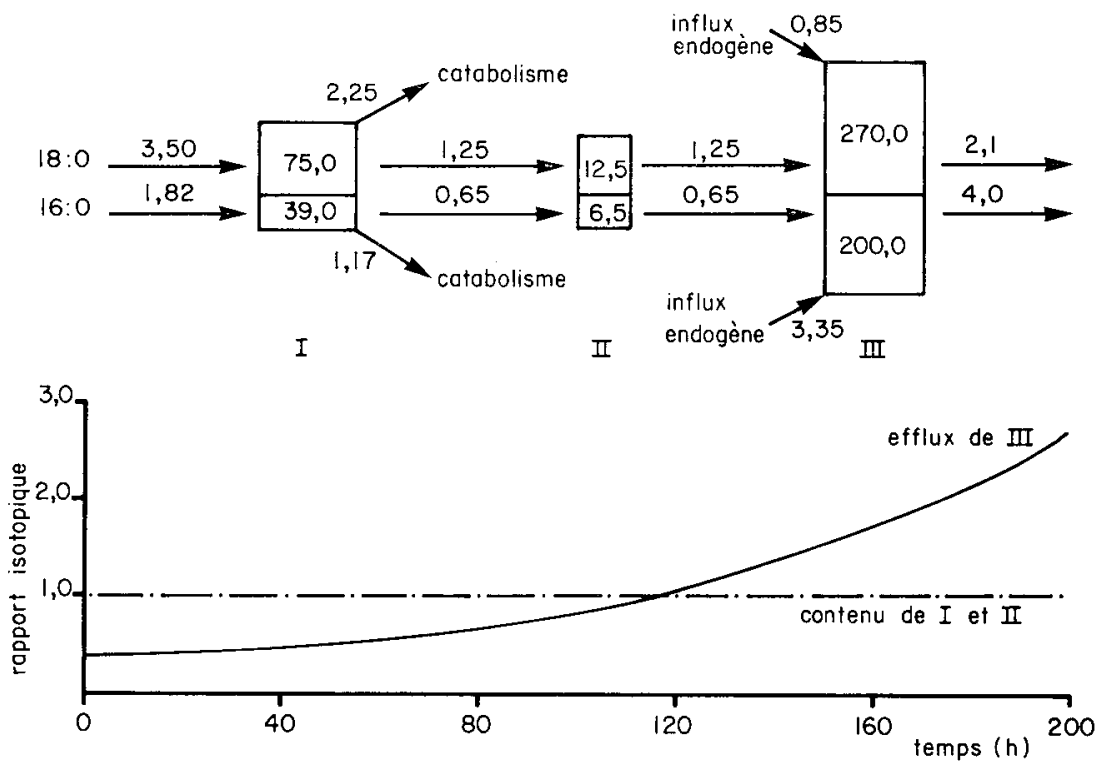

FIG. 6. - Modèle pour l'éfude de l'effet d'un influx d'acides gras non marqués dans la glande mammaire, sur l'évolution du rapport isotopique dans les lipides du lait (voir texte). Compartiments I, II et III représentent les acides gras triglycéridiques aux niveaux respectivement des lipides alimentaires, des VLDL et des lipides du lait. Les valeurs indiquées dans les compartiments expriment leurs tailles en grammes et celles sur les flèches expriment les flux en $\mathrm{g} / \mathrm{h}$. 
marqués par des acides endogènes non marqués dans la glande mammaire pourrait à elle seule rendre compte de la divergence entre les courbes exprimant le rapport isotopique dans les lipides du lait et dans leur précurseur (fig. 5). Si le rapport dans le précurseur restait constant ef ne diminuait pas, comme il le fait pendant la première moitié de l'expérience, on s'attendrait à voir une augmentation régulière du rapport dans le lait dès le début comme dans le modèle. Celui-ci, qui ne représente évidemment pas une notion, même très simplifiée, de l'organisation des fonds communs lipidiques de la vache, n'explique pas cette diminution du rapport dans le précurseur ; mais il nous semble du moins possible qu'un influx inégal d'acides non marqués du tube digestif vers le sife de formation des VLDL puisse en être responsable.

\section{L'acide phytanique.}

L'acide phytanique dérive par hydrogenation puis oxydation du phytol, alcool à 20 carbones estérifié dans la molécule de la chlorophylle, et il est donc un constituant normal des digesta. Comme on sait que la teneur des lipides animaux en cet acide est très variable (Duncan et Garton, 1963 ; Stead ef Welch, 1975 ; Patton et Benson, 1966) on peut se demander si la perméabilité des parois du tube digestif pourrait être influencée par les conditions externes y compris le stress. Dans ce cas une absorption accrue du 20:0 $0_{\text {ra }}$ suivie de son incorporation dans les triglycérides lipoprotéiques, presque irréversible à cause de la résistance bien connue de ces esters à la lipolyse, pourrait conduire à son accumulation.

Nous avons déjà mentionné l'hypothèse selon laquelle les triglycérides contenant un résidu 20:0 ra ne servent pas de précurseur des lipides du lait. Cette hypothèse est appuyée par les mesures à partir desquelles les résultats portés sur la figure 2 ont été calculés : elles montrent qu'il n'y a pas de différence AV appréciable en ces triglycérides. Elle est appuyée aussi par l'observation (Needs et Welch, résultats non publiés) que ces triglycérides ne sont guère hydrolysés par la lipoprotéinelipase alors que des triglycérides exempts de 20:0 ra le sont complètement.

De ce travail on peut tirer certaines conclusions positives. En premier lieu il est évident que tous les acides gras non ramifiés renfermés dans le précurseur sérique sont incorporés dans les lipides du lait dans la même proportion qu'ils se présentent à la glande mammaire. En l'absence de l'acide 20:0 ra on peut donc valablement utiliser les acides 16:0 ou 18:0 marqués pour mesurer la proportion des lipides du lait provenant du précurseur. Comme il est impossible d'assurer l'absence du 20:0 $\mathrm{ra}$ il est recommendable de s'en tenir au 16:0 marqué pour effectuer cette mesure. II apparaît que l'utilisation de deux isotopes pour étudier le métabolisme de deux composés différents ne constitue pas un système efficace pour la délection d'un fractionnement éventuel car une dilution différentielle, qui, elle-même, ne découle pas d'un fractionnement proprement dit, est capable de perturber le rapport isotopique.

Reçu en ovril 1978.

Accepté en août 1978.

Remerciements. - Nous remercions M. H. L. Buttle pour l'implantation des canules, M. C. Bishop pour avoir bien voulu programmer le modèle sur ordinateur ef $M M$. E. C. Needs and G. A. Payne pour leur aide technique. 


\section{Références}

BISHOP C., DAVIES T., GLASCOCK R. F., WELCH V. A., 1969. Studies on the origin of milk fat. A further study of bovine serum lipoproteins and an estimation of their contribution to milk fat. Biochem. J., 113, 629-633.

BISHOP C., GLASCOCK R. F., NEWELL E. M., WELCH V. A., 1971. Determination of the specific radioactivity of fatty acids separated as their methyl esters by gas-liquid chromatography. J. Lipid Res., 12, 777-780.

BRUMBY P. E., WELCH V. A., 1970. Fractionation of bovine serum lipoproteins and their characterization by gradient gel electrophoresis. J. Dairy Res., 37, 121-128.

CHARTON A., FAYE P., HERVY A., BERNARD C., GUESLIN M., 1965. Estimation de la quantité de glucose utilisée par la mamelle de la brebis au cours des trois premiers mois de lactation. C. R. Soc. Biol. Paris, 159, 1509-1511.

DUNCAN W. R. H., GARTON G. A., 1963. Blood lipids. 3. Plasma lipids of the cow during pregnancy and lactation. Biochem. J., 89, 414-419.

GLASCOCK R. F., REINIUS L. R., 1956. Studies on the origin of milk fat. 1. The location of tritium in stearic acid produced by the catalytic addition of tritium to elaidic acid. Biochem. J., 62, 529. 534.

GLASCOCK R. F., WELCH V. A., 1974. Contribution of the fatty acids of three low density serum lipoproteins to bovine milk fat. J. Dairy Sci., 57, 1364-1370.

GLASCOCK R. F., WELCH V. A., BISHOP C., DAVIES T., WRIGHT E. W., NOBLE R. C., 1966. An investigation of serum lipoproteins and of their contribution to milk fat in the dairy cow. Biochem. J., 98, 149-156.

PATTON S., BENSON A. A., 1966. Phytol metabolism in the bovine. Biochim. biophys. Acta, 125, 22-32.

STEAD D., WELCH V. A., 1975. Lipid composition of bovine serum lipoproteins. J. Dairy Sci., 58, 122-127. 